\title{
The significance of combining VEGFA, FLTI, and $K D R$ expressions in colon cancer patient prognosis and predicting response to bevacizumab
}

This article was published in the following Dove Press journal:

OncoTargets and Therapy

I5 April 2015

Number of times this article has been viewed

\author{
Shu-Dong Zhang ${ }^{1,2, *}$ \\ Cian M McCrudden ${ }^{3} *$ \\ Chen Meng ${ }^{4}$ \\ Yao $\operatorname{Lin}^{4}$ \\ Hang Fai Kwok ${ }^{1,3}$
}

'Faculty of Health Sciences, University of Macau, Avenida de Universidade, Macau, Special Administrative Region of the People's Republic of China; ${ }^{2}$ Center for Cancer Research and Cell Biology, Queen's University Belfast, United Kingdom; ${ }^{3}$ School of Pharmacy, Queen's University Belfast, United Kingdom; ${ }^{4}$ College of Life Sciences, Fujian Normal University, Fujian, People's Republic of China

*These authors contributed equally to this work

\begin{abstract}
Targeting angiogenesis through inhibition of the vascular endothelial growth factor (VEGF) pathway has been successful in the treatment of late stage colorectal cancer. However, not all patients benefit from inhibition of VEGF. Ras status is a powerful biomarker for response to anti-epidermal growth factor receptor therapy; however, an appropriate biomarker for response to anti-VEGF therapy is yet to be identified. VEGF and its receptors, FLT1 and KDR, play a crucial role in colon cancer progression; individually, these factors have been shown to be prognostic in colon cancer; however, expression of none of these factors alone was predictive of tumor response to anti-VEGF therapy. In the present study, we analyzed the expression levels of VEGFA, FLT1, and KDR in two independent colon cancer datasets and found that high expression levels of all three factors afforded a very poor prognosis. The observation was further confirmed in another independent colon cancer dataset, wherein high levels of expression of this three-gene signature was predictive of poor prognosis in patients with proficient mismatch repair a wild-type KRas status, or mutant p53 status. Most importantly, this signature also predicted tumor response to bevacizumab, an antibody targeting $V E G F A$, in a cohort of bevacizumab-treated patients. Since bevacizumab has been proven to be an important drug in the treatment of advanced stage colon cancer, our results suggest that the three-gene signature approach is valuable in terms of its prognostic value, and that it should be further evaluated in a prospective clinical trial to investigate its predictive value to anti-VEGF treatment.
\end{abstract}

Keywords: colon cancer, VEGFA, FLT1, KDR, angiogenesis, survival

\section{Introduction}

Angiogenesis plays a critical role in the progression of colon cancer, and the vascular endothelial growth factor (VEGF) pathway has been shown to play a central role in controlling tumor angiogenesis. ${ }^{1}$ VEGF ligands, such as $V E G F A, V E G F B, V E G F C$, and $V E G F D$ have different affinities toward, and activation potencies at, different VEGF receptors, including VEGFR1 (FLT1), VEGFR2 (KDR), and VEGFR3 (FLT4). ${ }^{2}$ $V E G F A$ has been shown to activate both FLT1 and $K D R,{ }^{2}$ and activation of this signaling pathway has led to vascularization and angiogenesis in malignant tissue to support its growth and survival. ${ }^{3} V E G F A$ has been shown to be overexpressed in colorectal cancer specimens, especially in those tumors with secondary metastases, including liver or lymph node metastases. ${ }^{4-7}$ Its mRNA expression in tumor as well as plasma is higher in colorectal cancer patients than in non-diseased patients. ${ }^{8}$ Despite this, VEGF expression has not been shown to be predictive of response to bevacizumab. ${ }^{9}$ In addition, VEGFA was shown to be a significant predictor for morbidity in a colorectal cancer cohort. ${ }^{10}$ 
Expression of FLT1, also known as VEGFR1, was shown to be higher in primary tumors and nodal metastases of colorectal carcinomas, and its overexpression was associated with lymphovascular invasion and local disease recurrence, but not with survival. ${ }^{11}$ Moreover, the serum level of soluble FLT1 has also been shown to be significantly higher in patients with colorectal cancer compared to non-diseased individuals. ${ }^{12}$ Likewise, $K D R$, also known as VEGFR2, has been shown to be overexpressed in metastatic colon cancer. ${ }^{6}$ Overexpression of its phosphorylated version was associated with larger tumor diameter and poor histological differentiation, ${ }^{10}$ while several single nucleotide polymorphisms of $K D R$ have also been identified that were postulated to modulate recurrence of colorectal cancer in patients receiving chemotherapy. ${ }^{13}$ Further investigation on various angiogenic factors in a large colorectal cancer patient cohort has further suggested that the expression of angiogenic factors can be associated with tumors of more aggressive phenotypes. ${ }^{14}$

Inhibition of the VEGF pathway using antibodies, recombinant fusion proteins, or tyrosine kinase inhibitors has been shown to be a successful approach in prolonging survival of patients with stage IV colorectal cancer. ${ }^{15}$ Bevacizumab is a humanized monoclonal immunoglobulin $\mathrm{G} 1$ antibody against soluble VEGFA that has been shown to be an effective and tolerable therapy that improves survival in colorectal cancer patients. ${ }^{16}$ In a phase III trial (AVF2107g), patients with metastatic colon cancer who received bevacizumab in addition to chemotherapy (irinotecan, bolus fluorouracil, and leucovorin) had significantly longer progression-free and overall survivals than patients who received chemotherapy only. ${ }^{17}$ Another phase III trial has also confirmed the efficacy of bevacizumab in treatment of colorectal cancer. ${ }^{18}$ Recent advances in the targeting of angiogenesis in colorectal cancer include the approval of novel agents, ${ }^{19,20}$ the demonstration of the importance of continuous inhibition of the VEGF pathway, ${ }^{19,21,22}$ and the investigation of the efficacy and safety of bevacizumab in the elderly. ${ }^{23}$ While the success of anti-EGFR (epidermal growth factor receptor) antibody therapy in advanced stage colorectal cancer patients has been improved by selecting patients based on their NRas status in addition to their KRas status, ${ }^{24-26}$ no such predictive biomarker exists for improving the success of antibody targeting of angiogenesis.

The prognostic value of individual angiogenic factors in colorectal cancer has been limited; for more comprehensive prognostication, co-consideration of multiple angiogenic factors and receptors may be more valuable. ${ }^{5}$ The survival rate of colorectal cancer patients was shown to be poor when their tumor cells overexpressed VEGFA, and their tumor-associated endothelial cells overexpressed $K D R .^{7}$ Antiangiogenic therapy is also a successful strategy in the treatment of lung cancer patients; $;^{27,28}$ we recently reported that a VEGFA-FLT1-KDR gene signature had strong prognostic significance in lung cancer patients. ${ }^{29}$ In the present study, we investigated the prognostic significance of the combined mRNA expressions of VEGFA, FLT1, and KDR in colorectal cancer using colon cancer datasets available in the Gene Expression Omnibus (GEO) database.

\section{Materials and methods Extraction of clinical and microarray gene expression data from colon cancer patient datasets}

Three colorectal cancer patient datasets, GSE14333, ${ }^{30}$ GSE14538, ${ }^{31}$ and GSE40967 $7^{32}$ were identified in the GEO database; datasets compiled using the HG-U133 microarray platform, which comprised $\geq 200$ patients for whom survival data were available in the GEO database were included in this study. A separate colorectal cancer patient cohort, GSE19862, which contained information on patient response to bevacizumab treatment, was also analyzed. Microarray gene expression data were retrieved from the data matrixes deposited to the GEO database by the original authors. R scripting was used to extract the expression values of a small number of genes (probesets) of interest, and the clinical data from the data matrixes were downloaded from GEO, as previously described. ${ }^{33}$

\section{Correlations of gene expression levels and clinical data}

All statistical analyses were performed using SPSS version 19.0. Expression levels were divided into high and low levels using the median expression level as the cut-off point for Kaplan-Meier survival analysis. Results were compared by log-rank test. Univariate Cox regression analysis was used to correlate gene expression levels and patient survival. Patients were divided into three groups based on the expression levels of VEGFA and FLT1; the VEGFA-FLT1-low group consisted of patients who expressed both genes below median levels (low); the VEGFA-FLT1-high group consisted of patients who expressed both genes at above median levels (high); the VEGFA-FLT1-intermediate group consisted of the remaining patients, who expressed either gene at above median level, and the other below median. Similar stratification was performed for the VEGFA-KDR grouping. Patients were also divided into four groups based on the expression levels of $V E G F A, F L T 1$, and KDR depending on the number of genes expressed at above the median. The survival time of patients 
stratified by this grouping method were analyzed by KaplanMeier analysis and Cox regression.

\section{Results}

The association between mRNA expression of VEGFA, FLTI, and KDR, and colon cancer patient survival

A high level of expression of VEGFA in both GSE14333 $(P=0.052)$ and $\operatorname{GSE} 17538(P=0.006)$ was associated with poorer disease-free survival (Figure 1A and B). Similarly, a high level of expression of FLT1 (VEGFR1) was associated

\section{A}

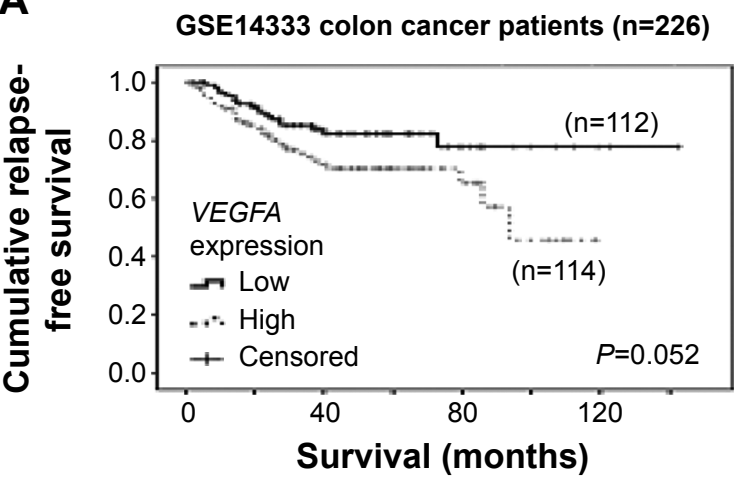

C
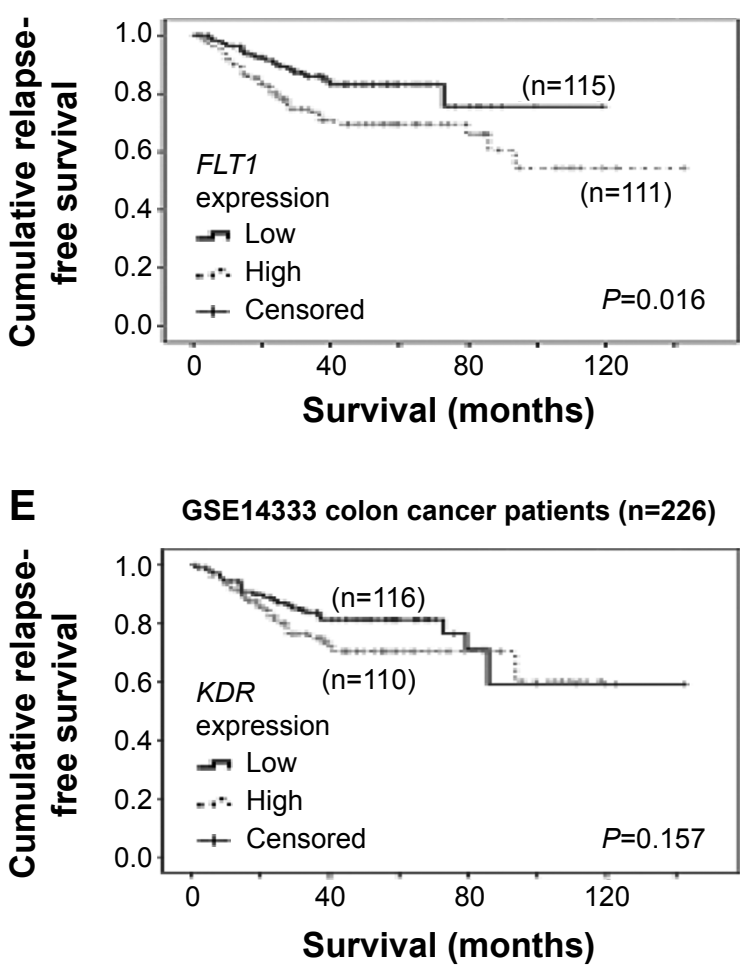

with poorer disease-free survival in both cohorts $(P=0.016$ for GSE14333 and $P=0.075$ for GSE17538; Figure 1C and D). However, the association between KDR (VEGFR2) expression and disease-free survival was less pronounced in these two datasets (Figure 1E and F).

\section{The prognostic significance of VEGFA- FLTI and VEGFA-KDR combinations}

The prognostic significance of $V E G F A, F L T 1$, or KDR was not consistently significantly demonstrated in the two colon cancer datasets. In an attempt to boost their prognostic power,
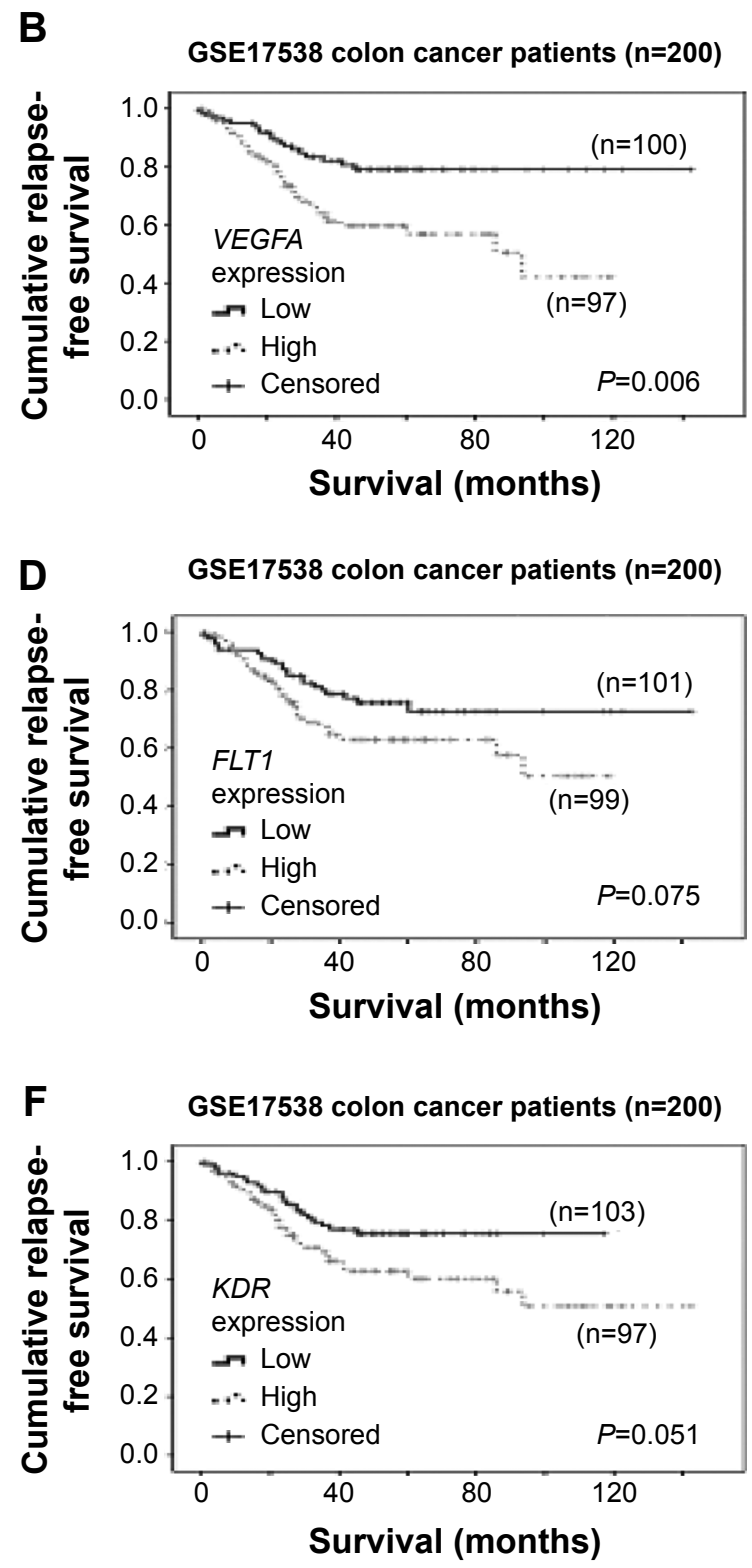

Figure I The association between VEGFA, FLTI, and KDR mRNA expression and colon cancer patient survival.

Notes: Kaplan-Meier analyses for VEGFA mRNA expression in (A) GSEI4333 and (B) GSEI7538. Kaplan-Meier analyses for FLTI mRNA expression in (C) GSEI4333 and (D) GSEI 7538. Kaplan-Meier analyses for KDR mRNA expression in (E) GSEI4333 and (F) GSEI7538.

Abbreviation: VEGFA, vascular endothelial growth factor a. 
we assessed co-overexpression of the ligand, $V E G F A$, and its receptors, FLT1 or KDR. As expected, patients whose primary tumors expressed high levels of both VEGFA and FLT1 had a significantly poorer survival than patients who had low expression of both VEGFA and FLT1 in both colon cancer datasets ( $P=0.008$ and $P=0.004$, respectively; comparison for all three groups, $P=0.004$ and $P=0.026$, respectively, for GSE14333 and GSE17538; Figure 2A and B). Similarly, patients whose primary tumors expressed high levels of both VEGFA and $K D R$ had a significantly poorer survival than patients who had low expression of both $V E G F A$ and $K D R$ in both colon cancer datasets $(P=0.011$ and $P=0.001$, respectively; comparison for all three groups, $P=0.053$ and $P=0.008$, respectively, for GSE14333 and GSE17538; Figure 2C and D).

\section{The prognostic significance of the VEGFA-FLTI-KDR combination}

Since the combinations of VEGFA-FLT1 and VEGFA-KDR were shown to be more consistent in terms of prognostication

A

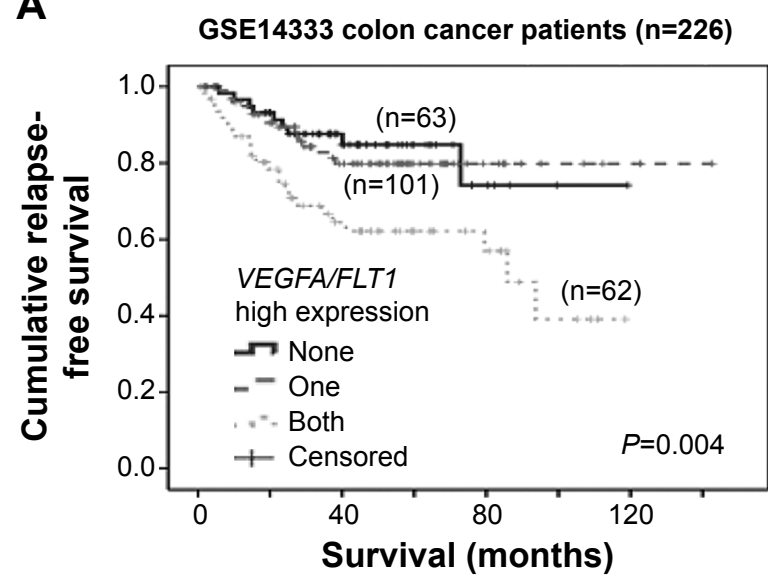

C

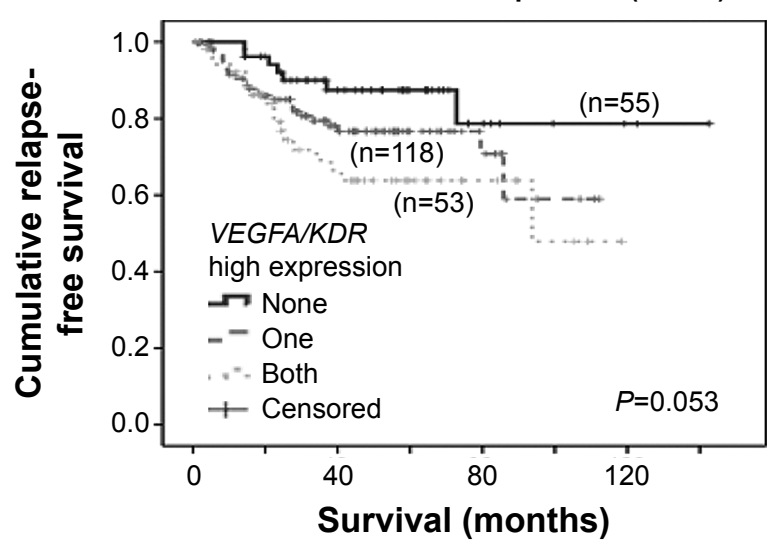

than when these genes were considered individually, we further investigated whether combination assessment of expression of the three genes, VEGFA-FLT1-KDR, could be a better prognostic indicator. In GSE14333, patients whose tumors expressed all three genes at a low level had a mean disease-free survival of 101 months (95\% confidence interval $[\mathrm{CI}]=86-116$ months), which is significantly longer than patients whose tumors expressed all three genes at a high level (mean disease-free survival $=72$ months, 95\% $\mathrm{CI}=54-90$ months). As shown in Figure 3A, the VEGFAFLT1-KDR gene signature was significantly associated with disease-free survival in colon cancer patients $(P=0.014)$. Similar results were obtained using the GSE17538 dataset. Patients whose tumors expressed all three genes at a low level had a mean disease-free survival of 104 months $(95 \% \mathrm{CI}=$ 95-113 months), while those who expressed all three genes at a high level had a mean disease-free survival of 71 months (95\% CI $=57-86$ months). As shown in Figure 3B, the VEGFA-FLT1-KDR gene signature was again significantly

B
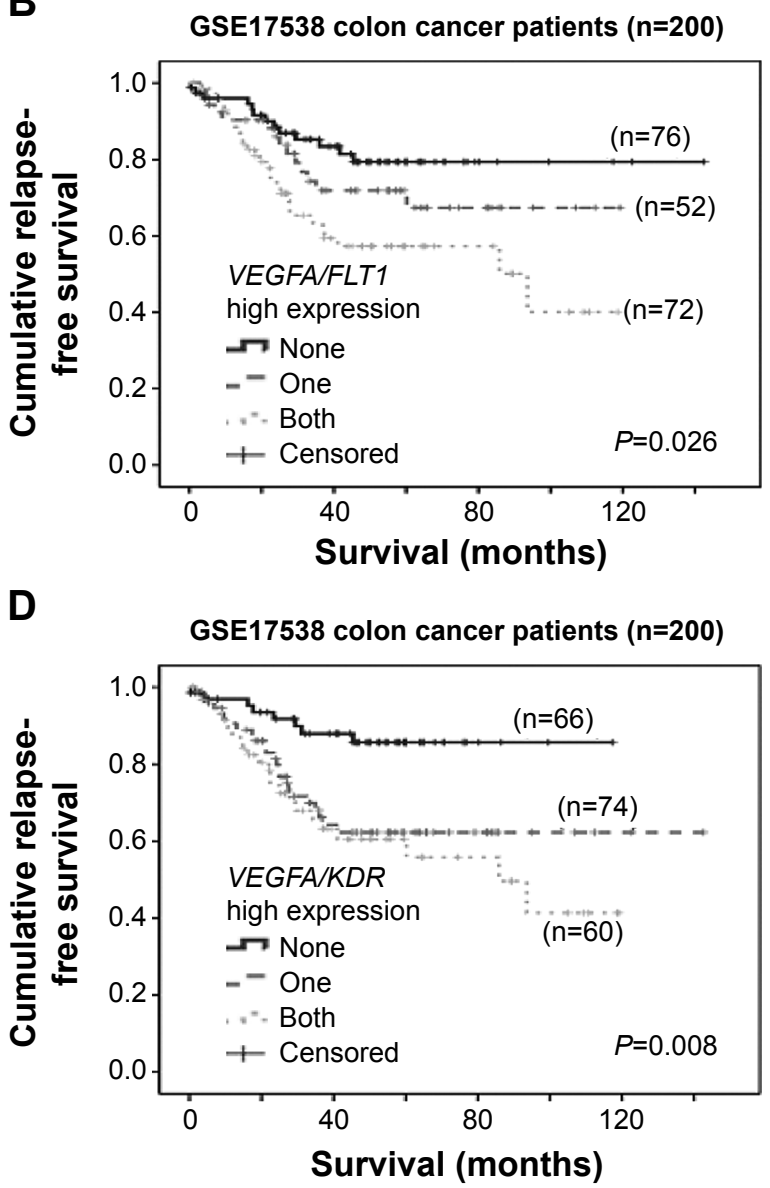

Figure 2 The association between combination of VEGFA/FLTI and VEGFA-KDR mRNA expression and colon cancer patient survival.

Notes: Kaplan-Meier analyses for VEGFA and FLTI mRNA expression in combination in (A) GSEI 4333 and (B) GSEI 7538. Kaplan-Meier analyses for VEGFA and KDR mRNA expression in combination in (C) GSEI4333 and (D) GSEI7538.

Abbreviation: VEGFA, vascular endothelial growth factor a. 

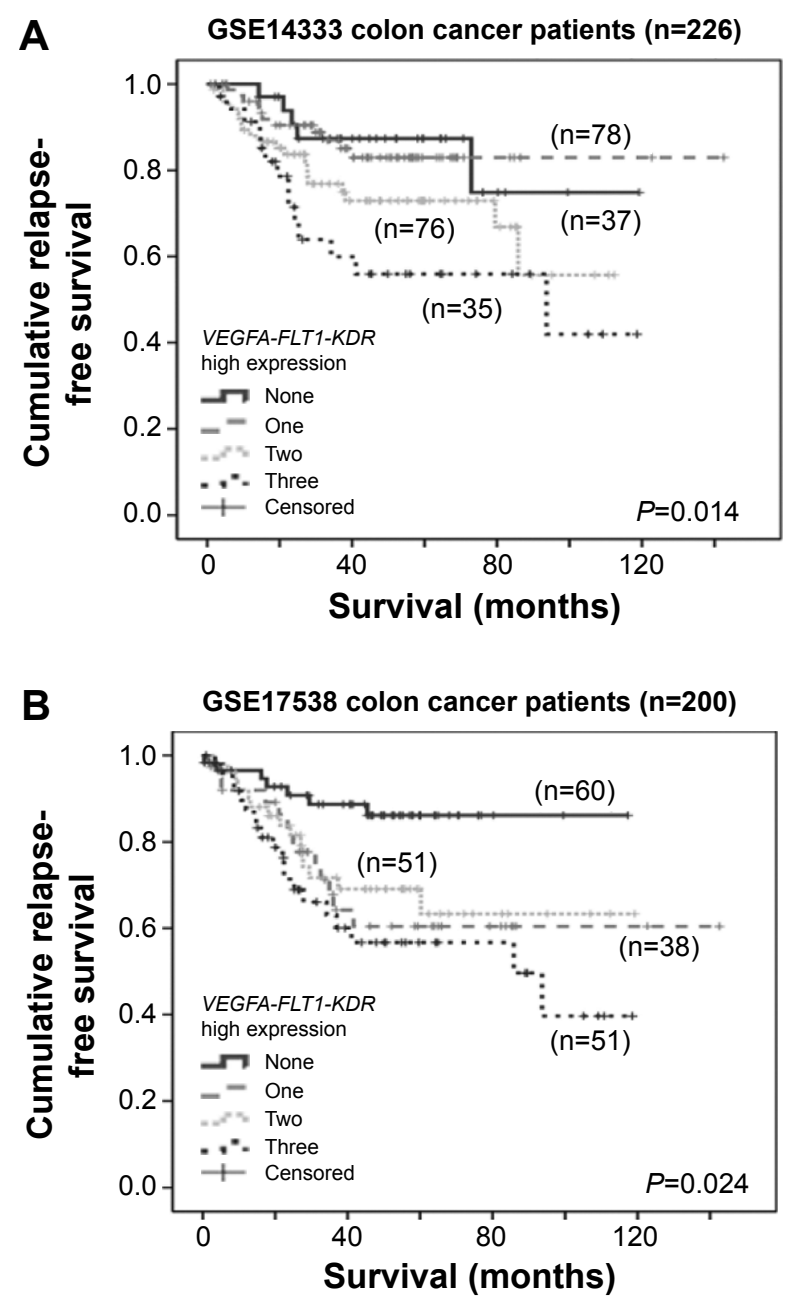

Figure 3 The association between the combination of VEGFA/FLTI/KDR mRNA expression and colon cancer patient survival.

Notes: Kaplan-Meier analyses for VEGFA, FLTI, and KDR mRNA expression in combination in (A) GSEI4333 and (B) GSEI7538.

Abbreviation: VEGFA, vascular endothelial growth factor a.

associated with disease-free survival in this patient cohort $(P=0.024)$.

\section{The impact of genetic alterations on the prognostic significance of the VEGFA- \\ FLTI-KDR combination}

In another independent dataset, GSE40967, which has available clinicopathological parameters, we sought to investigate whether the association between the three-gene signature and disease-free survival retained relevance in patients with specific genetic alterations. The clinical characteristics for patients in this cohort are listed in Table 1. In the GSE40967 colon cancer patient cohort, patients whose tumors had a high level of expression of the three-gene signature again had a significantly shorter disease-free survival than those patients whose tumors had a low level of
Table I Patient clinical and pathologic features for GSE40967

\begin{tabular}{llll}
\hline & $\begin{array}{l}\text { Number } \\
\text { of cases }\end{array}$ & Percent & $\begin{array}{l}\text { Median } \\
\text { (range) }\end{array}$ \\
\hline Age & 565 & & 68.I (22-97) \\
years
\end{tabular}

Abbreviations: MMR, mismatch repair; dMMR, deficient mismatch repair.

expression of the three-gene signature ( $P=0.048$; Figure 4A). Interestingly, this $V E G F A-F L T 1-K D R$ signature and survival association was only significant in patients with proficient mismatch repair (MMR) status $(P=0.031$; Figure 4B), but not in those with deficient MMR status ( $P=0.657$; data not shown). Likewise, the three-gene signature was a potent prognostic indicator in patients with wild-type KRas status $(P=0.001$; Figure $4 \mathrm{C})$, but not in those with mutant KRas status ( $P=0.848$; data not shown). Similarly, the three-gene signature was also significantly associated with survival in patients with mutant p53 status $(P=0.038$; Figure 4D), but 
A
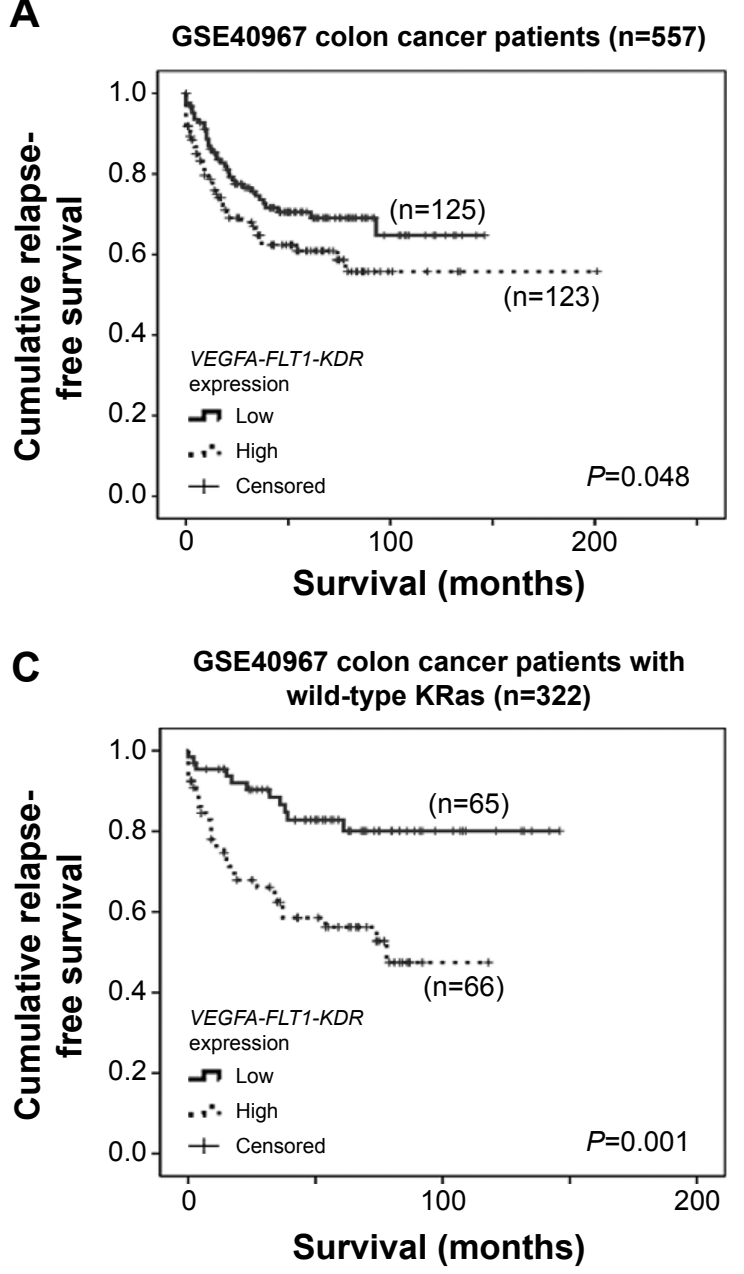

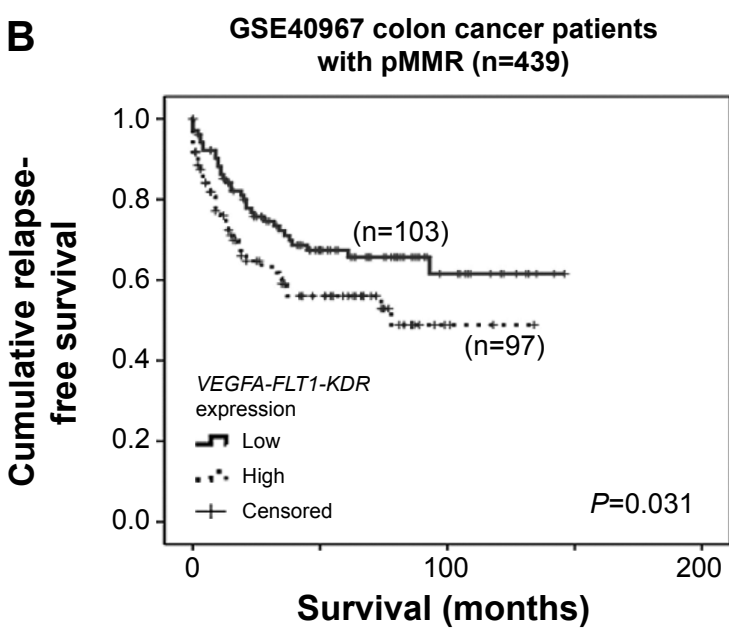

D GSE40967 colon cancer patients with mutant p53 (n=156)

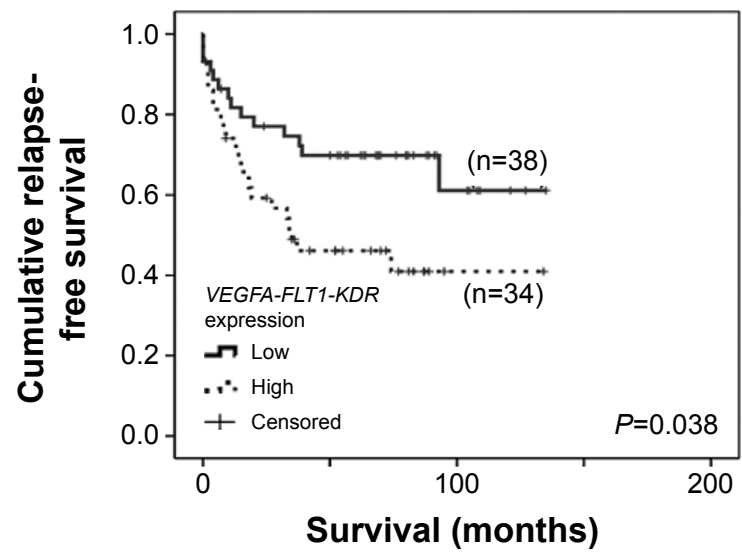

Figure 4 The association between the combination of VEGFA-FLTI-KDR mRNA expression and colon cancer patient survival in cohorts stratified based on genetic aberration.

Notes: Kaplan-Meier analyses for VEGFA, FLTI, and KDR mRNA expression in combination in GSE40967 in (A) the whole cohort, (B) patients with proficient mismatch repair, (C) patients with wild-type KRas status, and (D) patients with mutant p53 status.

Abbreviations: VEGFA, vascular endothelial growth factor a; mRNA, messenger ribonucleic acid; pMMR, proficient mismatch repair.

not in those with wild-type p53 status $(P=0.501$; data not shown).

\section{The predictive value of the three-gene signature to treatment of bevacizumab}

The ability of the three-gene signature to predict response to bevacizumab was tested in a cohort of colon cancer patients who had received bevacizumab treatment and for whom data regarding response to the therapy were known. We found that none of the patients who responded well to bevacizumab expressed the three-gene signature at a low level, while $71 \%$ of those patients who did not respond to bevacizumab expressed the three-gene signature at a low level; however only $14 \%$ of patients who did not respond to bevacizumab expressed this three-gene signature at a low level (chi-square test, $P=0.02$; Figure 5).

\section{Discussion}

Anti-angiogenic agents, such as bevacizumab, aflibercept, and regorafenib play an important role in the treatment of colon cancer. Importantly, continuous inhibition of the VEGF pathway has been shown to prolong survival in colon cancer patients, ${ }^{19-21}$ further reinforcing angiogenesis as an essential process in tumor progression and validating angiogenesis as an appropriate target for cancer therapy. Recent advances in molecular testing have led to the identification of a subgroup of patients, whose tumors have KRas or NRas mutations, for whom anti-EGFR antibodies, including cetuximab and panitumumab, are not a viable treatment option. ${ }^{24,34}$ However, no advances to identify suitable (or unsuitable) candidates for anti-angiogenesis treatment have been made. Our previous report showed that combining VEGFA, FLT1, and KDR expression could be a better prognostic indicator in lung 


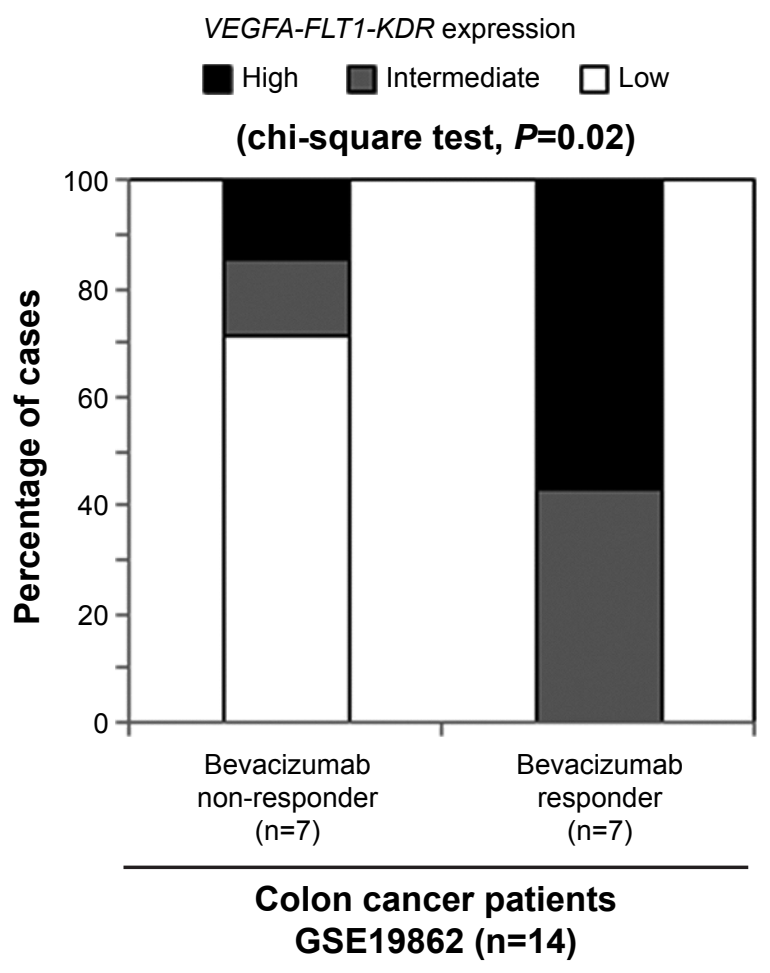

Figure $\mathbf{5}$ The association between the combination of VEGFA-FLTI-KDR mRNA expression and response to bevacizumab in colon cancer patients in the GSEI9862 dataset.

Abbreviation: VEGFA, vascular endothelial growth factor a.

cancer compared to when these factors were considered individually. ${ }^{29}$ In the present study, we have shown that using similar methodology, this three-gene signature is a robust prognostic indicator in colon cancer. Most importantly, this three-gene signature has been shown to predict the response to bevacizumab, an anti-angiogenic agent. Since VEGFA activates both $F L T 1$ and $K D R,{ }^{2}$ the co-overexpression of the ligand and the two receptors may be required for the activation of the pathway. In addition, as shown in Figure 3, the consideration of all three genes together can better stratify patients into smaller groups with a better defined prognosis, such that patients with all three genes expressed at high levels had the poorest prognosis compared to those with all three genes expressed at a low level, who had the best prognosis. Our results warrant further study on combining VEGFA, $F L T 1$, and $K D R$ expression profiling as a tool for predicting of response to anti-angiogenic agents in colorectal cancer patients. Since patient treatment was heterogeneous in the patient cohorts investigated in the present study, a prospective clinical trial is needed to test the clinical applicability for the three-gene signature as a prognostic and predictive biomarker for colorectal cancer patients.

Our results on the impact of genetic background on the prognostic significance of this three-gene signature for colon cancer are interesting and should be further investigated. MMR deficiency is an important prognostic determinant for colon cancer. ${ }^{35}$ A recent study has shown that bevacizumab may impose different effects on patients with MMR-deficient tumors compared to MMR-proficient tumors, ${ }^{36}$ in the current study, we show that the three-gene signature significantly predicted survival in patients with tumors proficient in MMR, but not in those with tumors deficient in MMR.

KRas status is an important biomarker for anti-EGFR therapy; however, it failed to offer predictive value for the anti-angiogenic agent, bevacizumab. ${ }^{37}$ As anti-EGFR agents are ineffective in colon cancers bearing KRas mutations, anti-angiogenic agents are the most common treatment option for this type of patients. In the present study, we found that the three-gene signature was highly prognostic in patients with wild-type KRas, but not in those with KRas mutation. Bevacizumab and cetuximab have both been shown to have similar efficacy in patients with KRas wild-type tumors in terms of response rate, progression-free survival, and overall survival, ${ }^{38-40}$ suggesting both anti-VEGF or anti-EGFR are viable options for colon cancer patients with wild-type KRas. The three-gene signature, when verified in a prospective trial, may be able to further stratify colon cancer patients with wild-type KRas in the future.

Previous reports investigating the association between mutational status of $\mathrm{p} 53$ and response to bevacizumab were contradictory; some reports showed that p53 status did not affect the efficacy of bevacizumab, ${ }^{41,42}$ while one report showed that patients with p53-positive (expression) tumors had a significantly longer survival when treated with bevacizumab with chemotherapy; ${ }^{43}$ another report showed that the progression-free survival was significantly longer with bevacizumab-containing regimens in patients with mutant p53 tumors, but not in those with wild-type p53 tumors. ${ }^{44}$ In the present study, our results show that the three-gene signature is prognostic only in tumors with mutant $\mathrm{p} 53$, but not in those with wild-type $\mathrm{p} 53$, suggesting that VEGFAmediated angiogenesis may play an important role in a p53 mutant genetic background. Further analysis is required to confirm the prognostic and predictive value of p53 in colon cancers treated with bevacizumab.

\section{Conclusion}

In conclusion, our results suggest that the three-gene signature may be a potential prognostic indictor for colon cancer and a potential predictive biomarker for response to anti-angiogenic agents such as bevacizumab. Importantly, the prognostic significance of this three-gene signature 
has also been demonstrated in lung cancer, in which the efficacy of anti-angiogenic agents has been shown. Since anti-angiogenic agents have been shown to be active also in other cancer types, further investigation into the prognostic significance of this three-gene signature in other types of cancer is warranted.

\section{Acknowledgments}

This study was supported by the University of Macau Start-Up Research Grant (SRG2015-00006-FHS) and MultiYear Research Grant (MYRG2015-00065-FHS), the National Science Foundation for Young Scientists of China (Grant No 31301172), and the Natural Science Foundation of Fujian Province (Grant No 2014J01122). We would like to thank Omic Science and Technology Limited for supporting the gene analysis in this study.

\section{Disclosure}

The authors report no conflicts of interest in this work.

\section{References}

1. Rmali KA, Puntis MC, Jiang WG. Tumour-associated angiogenesis in human colorectal cancer. Colorectal Dis. 2007;9(1):3-14.

2. Takahashi S. Vascular endothelial growth factor (VEGF), VEGF receptors and their inhibitors for antiangiogenic tumor therapy. Biol Pharm Bull. 2011;34(12):1785-1788.

3. Chekhonin VP, Shein SA, Korchagina AA, Gurina OI. VEGF in tumor progression and targeted therapy. Curr Cancer Drug Targets. 2013; 13(4):423-443.

4. George ML, Tutton MG, Janssen F, etal. VEGF-A, VEGF-C, and VEGF-D in colorectal cancer progression. Neoplasia. 2001;3(5):420-427.

5. André T, Kotelevets L, Vaillant JC, et al. Vegf, Vegf-B, Vegf-C and their receptors KDR, FLT-1 and FLT-4 during the neoplastic progression of human colonic mucosa. Int J Cancer. 2000;86(2):174-181.

6. Takahashi Y, Kitadai Y, Bucana CD, Cleary KR, Ellis LM. Expression of vascular endothelial growth factor and its receptor, KDR, correlates with vascularity, metastasis, and proliferation of human colon cancer. Cancer Res. 1995;55(18):3964-3968.

7. Harada Y, Ogata Y, Shirouzu K. Expression of vascular endothelial growth factor and its receptor KDR (kinase domain-containing receptor)/Flk-1 (fetal liver kinase-1) as prognostic factors in human colorectal cancer. Int J Clin Oncol. 2001;6(5):221-228.

8. Garcia V, Garcia JM, Silva J, et al. Levels of VEGF-A mRNA in plasma from patients with colorectal carcinoma as possible surrogate marker of angiogenesis. J Cancer Res Clin Oncol. 2008;134(11): $1165-1171$.

9. Aoyagi Y, Inuma H, Horiuchi A, Shimada R, Watanabe T. Association of plasma VEGF-A, soluble VEGFR-1 and VEGFR-2 levels and clinical response and survival in advanced colorectal cancer patients receiving bevacizumab with modified FOLFOX6. Oncol Lett. 2010;1(2):253-259.

10. Giatromanolaki A, Koukourakis MI, Sivridis E, et al. Activated VEGFR2/KDR pathway in tumour cells and tumour associated vessels of colorectal cancer. Eur J Clin Invest. 2007;37(11):878-886.

11. Al-Maghrabi J, Gomaa W, Buhmeida A, Qari Y, Al-Qahtani M, Al-Ahwal M. Prognostic significance of VEGFR1/Flt-1 immunoexpression in colorectal carcinoma. Tumour Biol. 2014;35(9):9045-9051.
12. Abbasi O, Mashayekhi F, Mirzajani E, Fakhriyeh Asl S, Mahmoudi T, Saeedi Saedi H. The soluble VEGFR1 concentration in the serum of patients with colorectal cancer. Surg Today. 2015;45(2):215-220.

13. Dong G, Guo X, Fu X, et al. Potentially functional genetic variants in KDR gene as prognostic markers in patients with resected colorectal cancer. Cancer Sci. 2012;103(3):561-568.

14. Martins SF, Garcia EA, Luz MA, Pardal F, Rodrigues M, Filho AL. Clinicopathological correlation and prognostic significance of VEGF-A, VEGF-C, VEGFR-2 and VEGFR-3 expression in colorectal cancer. Cancer Genomics Proteomics. 2013;10(2):55-67.

15. Grothey A, Galanis E. Targeting angiogenesis: progress with antiVEGF treatment with large molecules. Nat Rev Clin Oncol. 2009; 6(9):507-518.

16. Yeung Y, Tebbutt NC. Bevacizumab in colorectal cancer: current and future directions. Expert Rev Anticancer Ther. 2012;12(10):1263-1273.

17. Hurwitz H, Fehrenbacher L, Novotny W, et al. Bevacizumab plus irinotecan, fluorouracil, and leucovorin for metastatic colorectal cancer. N Engl J Med. 2004;350(23):2335-2342.

18. Saltz LB, Clarke S, Díaz-Rubio E, et al. Bevacizumab in combination with oxaliplatin-based chemotherapy as first-line therapy in metastatic colorectal cancer: a randomized phase III study. J Clin Oncol. 2008;26(12):2013-2019.

19. Van Cutsem E, Tabernero J, Lakomy R, et al. Addition of aflibercept to fluorouracil, leucovorin, and irinotecan improves survival in a phase III randomized trial in patients with metastatic colorectal cancer previously treated with an oxaliplatin-based regimen. J Clin Oncol. 2012;30(28):3499-3506.

20. Grothey A, Van Cutsem E, Sobrero A, et al. Regorafenib monotherapy for previously treated metastatic colorectal cancer (CORRECT): an international, multicentre, randomised, placebo-controlled, phase 3 trial. Lancet. 2013;381(9863):303-312.

21. Bennouna J, Sastre J, Arnold D, et al; ML18147 Study Investigators. Continuation of bevacizumab after first progression in metastatic colorectal cancer (ML18147): a randomised phase 3 trial. Lancet Oncol. 2013;14(1):29-37.

22. Kubicka S, Greil R, André T, et al; ML18147 study investigators including AIO, GERCOR, FFCD, UNICANCER GI, TTD, BGDO, GEMCAD, and AGMT groups. Bevacizumab plus chemotherapy continued beyond first progression in patients with metastatic colorectal cancer previously treated with bevacizumab plus chemotherapy: ML18147 study KRAS subgroup findings. Ann Oncol. 2013; 24(9):2342-2349.

23. Cunningham D, Lang I, Marcuello E, et al; AVEX study investigators. Bevacizumab plus capecitabine versus capecitabine alone in elderly patients with previously untreated metastatic colorectal cancer (AVEX): an open-label, randomised phase 3 trial. Lancet Oncol. 2013;14(11):1077-1085.

24. Douillard JY, Oliner KS, Siena S, et al. Panitumumab-FOLFOX4 treatment and RAS mutations in colorectal cancer. $N$ Engl J Med. 2013;369(11):1023-1034.

25. Amado RG, Wolf M, Peeters M, et al. Wild-type KRAS is required for panitumumab efficacy in patients with metastatic colorectal cancer. J Clin Oncol. 2008;26(10):1626-1634.

26. Van Cutsem E, Köhne CH, Láng I, et al. Cetuximab plus irinotecan, fluorouracil, and leucovorin as first-line treatment for metastatic colorectal cancer: updated analysis of overall survival according to tumor KRAS and BRAF mutation status. J Clin Oncol. 2011;29(15):2011-2019.

27. Sandler A, Gray R, Perry MC, et al. Paclitaxel-carboplatin alone or with bevacizumab for non-small-cell lung cancer. $N$ Engl J Med. 2006;355(24):2542-2550.

28. Reck M, Kaiser R, Mellemgaard A, et al; LUME-Lung 1 Study Group. Docetaxel plus nintedanib versus docetaxel plus placebo in patients with previously treated non-small-cell lung cancer (LUME-Lung 1): a phase 3, double-blind, randomised controlled trial. Lancet Oncol. 2014;15(2):143-155. 
29. Zhang S, McCrudden C, Kwok H. The prognostic significance of combining VEGFa, FLT1 and KDR mRNA expressions in lung cancer. Oncol Lett. 2015; in press.

30. Jorissen RN, Gibbs P, Christie M, et al. Metastasis-associated gene expression changes predict poor outcomes in patients with Dukes Stage B and C colorectal cancer. Clin Cancer Res. 2009;15(24):7642-7651.

31. Smith JJ, Deane NG, Wu F, et al. Experimentally derived metastasis gene expression profile predicts recurrence and death in patients with colon cancer. Gastroenterology. 2010;138(3):958-968.

32. Marisa L, de Reyniès A, Duval A, et al. Gene expression classification of colon cancer into molecular subtypes: characterization, validation, and prognostic value. PLoS Med. 2013;10(5):e1001453.

33. Yuen HF, Gunasekharan VK, Chan KK, et al. RanGTPase: a candidate for Myc-mediated cancer progression. J Natl Cancer Inst. 2013; 105(7):475-488.

34. Sorich MJ, Wiese MD, Rowland A, Kichenadasse G, McKinnon RA, Karapetis CS. Extended RAS mutations and anti-EGFR monoclonal antibody survival benefit in metastatic colorectal cancer: a meta-analysis of randomized, controlled trials. Ann Oncol. 2015;26(1):13-21.

35. Sinicrope FA, Mahoney MR, Smyrk TC, et al. Prognostic impact of deficient DNA mismatch repair in patients with stage III colon cancer from a randomized trial of FOLFOX-based adjuvant chemotherapy. J Clin Oncol. 2013;31(29):3664-3672.

36. Pogue-Geile K, Yothers G, Taniyama Y, et al. Defective mismatch repair and benefit from bevacizumab for colon cancer: findings from NSABP C-08. J Natl Cancer Inst. 2013;105(13):989-992.

37. Price TJ, Hardingham JE, Lee CK, et al. Impact of KRAS and BRAF gene mutation status on outcomes from the phase III AGITG MAX trial of capecitabine alone or in combination with bevacizumab and mitomycin in advanced colorectal cancer. J Clin Oncol. 2011;29(19):2675-2682.
38. Venook A, Niedzwiecki D, Lenz H. Phase III trial of irinotecan/5-FU/leucovorin (FOLFIRI) or oxaliplatin/5-FU/leucovorin (mFOLFOX6) with bevacizumab (BV) or cetuximab (CET) for patients (pts) with KRAS wild-type (wt) untreated metastatic adenocarcinoma of the colon or rectum (MCRC). Proc Am Soc Clin Oncol. 2014;32(Suppl 15):LBA3.

39. Petrelli F, Coinu A, Barni S. FOLFIRI with cetuximab or bevacizumab: FIRE-3. Lancet Oncol. 2014;15(13):e581-e582.

40. Heinemann V, von Weikersthal LF, Decker T, et al. FOLFIRI plus cetuximab versus FOLFIRI plus bevacizumab as first-line treatment for patients with metastatic colorectal cancer (FIRE-3): a randomised, open-label, phase 3 trial. Lancet Oncol. 2014;15(10):1065-1075.

41. Ince WL, Jubb AM, Holden SN, et al. Association of k-ras, b-raf, and p53 status with the treatment effect of bevacizumab. J Natl Cancer Inst. 2005;97(13):981-989.

42. Kara O, Duman BB, Kara B, Erdogan S, Parsak CK, Sakman G. Analysis of PTEN, VEGF, HER2 and P53 status in determining colorectal cancer benefit from bevacizumab therapy. Asian Pac J Cancer Prev. 2012;13(12):6397-6401.

43. Yang SX, Steinberg SM, Nguyen D, Swain SM. p53, HER2 and tumor cell apoptosis correlate with clinical outcome after neoadjuvant bevacizumab plus chemotherapy in breast cancer. Int J Oncol. 2011;38(5):1445-1452.

44. Said R, Hong DS, Warneke CL, et al. P53 mutations in advanced cancers: clinical characteristics, outcomes, and correlation between progression-free survival and bevacizumab-containing therapy. Oncotarget. 2013;4(5):705-714.
OncoTargets and Therapy

\section{Publish your work in this journal}

OncoTargets and Therapy is an international, peer-reviewed, open access journal focusing on the pathological basis of all cancers, potential targets for therapy and treatment protocols employed to improve the management of cancer patients. The journal also focuses on the impact of management programs and new therapeutic agents and protocols on

\section{Dovepress}

patient perspectives such as quality of life, adherence and satisfaction. The manuscript management system is completely online and includes a very quick and fair peer-review system, which is all easy to use. Visit http://www.dovepress.com/testimonials.php to read real quotes from published authors. 